DOI: https://doi.org/10.46296/rc.v5i9.0047

\title{
Durabilidad de la Mortadella Novel como contribución a la seguridad alimentaria local
}

\section{Durability of mortadela Novel as a contribution to local food security}

\author{
Matos-Mosqueda Luisa \\ Profesora Titular del departamento de Ciencia y Tecnología de los Alimentos, \\ Universidad de Camagüey Ignacio Agramonte Loynaz. Camagüey, Cuba. \\ Correo: luisa.matos@reduc.edu.cu \\ ORCID ID: http://orcid.org/0000-0002-2387-163X \\ León-Valle Wilson \\ Docente Investigador del Sistema de Educación Superior, \\ Universidad Estatal Península de Santa Elena. Santa Elena, Ecuador. \\ Correo: bleon@upse.edu.ec \\ ORCID ID: http://orcid.org/0000-0002-3374-5207
}

Benítez-Cortés Isnel

Profesor Titular del departamento de Ciencia y Tecnología de los Alimentos, Universidad de Camagüey Ignacio Agramonte Loynaz. Camagüey, Cuba. Correo: isnel.benites@reduc.edu.cu ORCID ID: https://orcid.org/0000-0003-2427-640X

\author{
Arias-Muñíz Dalgis \\ Estudiante de Licenciatura en Ciencias Alimentarias \\ Universidad de Camagüey Ignacio Agramonte Loynaz. Camagüey, Cuba. \\ Correo: dalgis.arias@reduc.edu.cu \\ Méndez-Pérez Daily \\ Técnica Analista de laboratorio, Unidad Empresarial de \\ Base Álvaro Barba Machado. Camagüey, Cuba. \\ Correo: daylimp479@gmail.com
}

\section{RESUMEN}

La presente investigación se realizó en la Unidad Empresarial de Base "Álvaro Barba Machado", del municipio Colombia, perteneciente a la Empresa Cárnica de Las Tunas. El objetivo de la misma fue: Estimar el tiempo de vida útil de la Mortadella Novel que se produce en el Combinado cárnico Álvaro Barba Machado como contribución a la seguridad alimentaria local. Se evaluaron cinco muestras del producto Mortadella Novel CRD 22 - CCD 33, lote 6, lo que se realizó a los $7,10,14,17$ y 20 días después de producida la mortadella. Para determinar la vida útil del producto se aplicó el método directo, a través del análisis de los resultados físico-químicos, microbiológicos y sensoriales aplicados al mismo, según lo establecido por la NRIAL 189:03 y los resultados se encontraron dentro de norma. Se realizó un análisis de varianza para los resultados químico-físicos, y no se encontraron diferencias significativas entre los lotes analizados. A través de los resultados sensoriales y químico - físicos, se detectó un punto de

\section{Información del manuscrito:}

Fecha de recepción: 01 de diciembre de 2021.

Fecha de aceptación: 27 de diciembre de 2021.

Fecha de publicación: 06 de enero de 2022. 
viraje en los mismos a los 14 días después de producida la Mortadella Novel, por lo que se estimó este, como el tiempo de vida útil para este producto.

Palabras claves: mortadella, vida útil, durabilidad, seguridad alimentaria local.

\begin{abstract}
This research was carried out at the "Álvaro Barba Machado" Base Business Unit, in the municipality of Colombia, belonging to the Las Tunas Meat Company. The objective of the same was: Estimate the lifetime of the Mortadella Novel that occurs in the Alvaro Barba Machado Meat Complex as a contribution to local food security. Five samples of the Mortadella Novel CRD 22 CCD 33 product, lot 6 were evaluated, which was done at 7, 10, 14, 17 and 20 days after the production of mortadella. To determine the shelf life of the product, the direct method was applied, through the analysis of the physical-chemical, microbiological and sensory results applied to it, as established by NRIAL 189: 03 and the results were within the norm. An analysis of variance was performed for the chemical-physical results, and no significant differences were found between the lots analyzed. Through the sensory and chemical - physical results, a turning point was detected in them at 14 days after the Mortadella Novel was produced, so it was estimated as the lifetime of this product.
\end{abstract}

Keywords: mortadella, shelf life, durability, local food security.

\title{
1. INTRODUCCIÓN
}

El desarrollo de la Industria Alimenticia cubana comenzó con el triunfo de la Revolución, momento en que se fueron agrupando las fábricas en sectores especializados y un ejemplo de ello es la industria cárnica. La producción de derivados cárnicos ocupa un rol importante en esta esfera, lo que se debe a la posición que ocupan estos productos en la escala de preferencias alimentarias, por su alto contenido en proteínas (Cali, Carolina, 2015). Las empresas cárnicas en el país trabajan con el objetivo de entregar productos a la canasta básica familiar para lograr así la satisfacción de las necesidades nutricionales y ofrecer una dieta balanceada a la población como pilar primordial para el logro de la seguridad alimentaria.

La industria cárnica cubana se encuentra en proceso de desarrollo, adoptando tecnologías, técnicas y procedimientos que le conlleven al incremento de indicadores económicos importantes como la eficiencia, la competitividad y reducir los costos de producción. Para ello, en dependencia de sus potencialidades de las empresas en cada localidad, se fomenta el desarrollo y mantenimiento de sus producciones, potenciando el uso de subproductos, el 
enriquecimiento de los productos mediante el uso de extensores y el aprovechamiento de los productos cárnicos en la elaboración de embutidos.

La mortadella constituye un embutido escaldado, compuesto por una emulsión de carne vacuna, carne de cerdo y gordura de cerdo finamente picada, que se mezcla con dados de tocino de cerdo en cubos y es embutido en tripas natural como la vejiga o sintéticas como celofán, fibrosa o poliamida (Peñaherrera, 2018). Posee un contenido relativamente bajo de sodio y se digiere fácilmente debido a la finura del relleno y los efectos de la cocción a la que se somete.

En el mundo actual, la cadena de producción y distribución de alimentos es cada vez más larga y en la mayoría de los casos el alimento llega hasta el consumidor luego de recorrer una serie de modificaciones y transformaciones (Da Silva, 2016).

En la industria cárnica y en todas las industrias de alimentos es imprescindible obtener productos con calidad, ya que de ello depende la seguridad alimentaria, aceptación por parte de los consumidores y que no existan riesgos para la salud. La determinación oportuna y objetiva de la vida útil permite a los empresarios evitar pérdidas por devolución, ampliar su mercado nacional y de exportación, y la confianza del consumidor. Además, cuando se lanza un nuevo producto al mercado, hay sustitución o cambio de especificaciones de alguna materia prima, se hace también necesario la determinación de la "vida útil" (Llamba y Jackeline, 2012).

La vida útil de un alimento se define como un periodo de tiempo en el que un producto sigue siendo seguro y cumple sus especificaciones de calidad en condiciones de almacenamiento y uso previstas. La vida útil determina la fecha de caducidad y se expresa como "fecha de caducidad" o "consumir preferentemente antes de" (Rubio, Español, Altolaguirre, Cabedo, Vázquez, Iñigo, Madrid, Fernandez, Palop, 2019).

\section{MATERIALES Y MÉTODOS}

El trabajo se realizó en la UEB Álvaro Barba Machado del municipio Colombia, y el producto que se evaluó fue la mortadella tipo Novel, elaborada en esta 
industria. Se determinó el tamaño de muestra para validar el estudio, y se consult con el Instituto de Investigaciones para la Industria Alimentaria (IIIA). Se concluyó que la muestra minima representativa, teniendo en cuenta las producciones en la entidad, es de cinco por lotes. El cronograma de muestreo que se estableció fue a los 7, 10, 14, 17 y 20 días de producida la mortadella tipo Novel, tomando en cuenta que el tiempo de vida útil establecido en la industria para este producto, según la NEIAL 2660-01: 2017 es de 7 días.

Para la toma de muestras se emplearon bolsas de nylon estériles, las cuales fueron analizadas en el Centro de Higiene y Epidemiología del municipio Amancio. Las mortadellas analizadas fueron las siguientes: mortadella Novel CRD 22 - CCD 33 (CRD: carne de res deshuesada; CCD: Carne de cerdo deshuesada). Luego de analizar estas determinaciones se procedió a calcular la vida útil del producto utiliando el método directo.

El control de temperatura durante el almacenamiento se realizó diariamente y en cada turno de trabajo. Se utilizó un plan de muestreo simple, de inspección normal, según lo establecido en la NC ISO 2859-1:2003 - Procedimiento de muestreo para la inspección por atributos parte 1: Esquemas de muestreo indexado por el nivel de calidad aceptable (NCA) para la inspección lote a lote. Las especificaciones físico - químicas y microbiológicas de este producto se consultaron en la NRIAL 189:03: Carne y productos cárnicos. Mortadella Novel. Especificaciones de calidad. Los ensayos correspondientes se realizaron según las normas cubanas.

\section{RESULTADOS}

La responsabilidad de la determinación de la vida útil es de los productores los cuales deben tener la capacidad de probar y evaluar la vida útil de los productos, además, la evaluación de la vida útil es parte de todo programa de desarrollo de productos Nuñez de Villavicencio, Rodríguez, Hernández, Rodríguez, 2017).

Según Nuñez de Villavicencio y col., un estudio de vida útil es un medio objetivo y metódico para determinar o estimar el tiempo que puede esperarse que se mantenga un producto alimenticio, sin cambios apreciables en su calidad y debe realizarse durante el desarrollo de nuevos productos o cuando se producen 
cambios en la formulación, en las condiciones de procesamiento, en los materiales de envase y composición de la atmósfera, de los suministradores de ingredientes o envases, en las condiciones de almacenamiento y durante el curso de una vigilancia regular de la vida útil.

Dentro de los parámetros de medición de vida útil, los microbiológicos tienen gran importancia, tomando en cuenta que determinan la inocuidad del producto o la agrupación de condiciones y medidas necesarias durante la producción, almacenamiento, distribución y preparación de alimentos para asegurar que una vez ingeridos, no representen un riesgo para la salud del consumidor (Aguilera, 2020).

Una de las industrias productoras y comercializadora de carnes y derivados cárnicos en Cuba es el Combinado Cárnico Álvaro Barba Machado, perteneciente a la Empresa Cárnica de Las Tunas. En dicha entidad se producen diferentes clases de embutidos, entre ellos la Mortadella Novel, producto destinado a canasta básica. Teniendo en cuenta que éste es un producto demandando por la población, se requiere una calidad que permita la aceptación y seguridad alimentaria de los consumidores. Aunque en la UEB se trabaja bajo los estándares de calidad requeridos en todas las líneas de producción, surge la necesidad de estimar el tiempo de vida útil real de la misma.

Es por ello que esta investigación se diseña para estimar el tiempo de vida útil de la Mortadella Novel que se produce en el Combinado Cárnico Álvaro Barba Machado, con el empleo del método directo, a través de la valoración físicoquímica, microbiológica y sensorial.

\subsection{Resultados de las valoraciones microbiológica y sensorial}

El estudio demostró que los indicadores microbiológicos cumplieron con la norma de calidad, no se detectó ningún proceso de contaminación, ni alteración del producto.

Atendiendo a los resultados presentados en la tabla 1, se denota la calificación de Buena hasta el día 14, al valorar el aspecto al corte se tiene presencia de pellejos y tendones ligera durante los 20 días de los ensayos. Sin embargo, los jueces observan a los 17 días defectos ligeros en cuanto a la dureza y dificultad 
en el rebanado, y a los 20 días este aspecto asciende su valoración a moderado, lo que indica que hay una tendencia al incremento de este defecto. Resultados estos que se muestran en la tabla.

Tabla 1. Resultados Sensoriales Mortadella Novel CRD 22 - CCD 33 Lote 6.

\begin{tabular}{|c|c|c|c|c|c|}
\hline \multirow[t]{2}{*}{ Producto } & \multicolumn{5}{|c|}{ Mortadella Novel CRD 22 - CCD 33 Lote 6} \\
\hline & 7 días & 10 días & 14 días & 17 días & 20 días \\
\hline $\begin{array}{l}\text { Puntuación } \\
\text { promedio }\end{array}$ & 24,0 & 24,0 & 24,0 & 22,0 & 21,0 \\
\hline $\begin{array}{ll}\text { Factor } & \text { de } \\
\text { conversión }\end{array}$ & \multicolumn{5}{|c|}{0,8} \\
\hline $\begin{array}{l}\text { Puntuación } \\
\text { convertida }\end{array}$ & 19,2 & 19,2 & 19,2 & 17,6 & 16,8 \\
\hline $\begin{array}{l}\text { Puntuación } \\
\text { total }\end{array}$ & 18,0 & 18,0 & 18,0 & 16,4 & 12,0 \\
\hline Calificación & Buena & Buena & Buena & Aceptable & Mala \\
\hline $\begin{array}{l}\text { Observacio- } \\
\text { nes }\end{array}$ & $\begin{array}{l}\text { Aspecto al } \\
\text { corte: } \\
\text { presencia } \\
\text { de pellejos } \\
\text { y tendones } \\
\text { (ligero) }\end{array}$ & $\begin{array}{l}\text { Aspecto al } \\
\text { corte: } \\
\text { presencia } \\
\text { de pellejos } \\
\text { y tendones } \\
\text { (ligero) }\end{array}$ & $\begin{array}{l}\text { Aspecto al } \\
\text { corte: } \\
\text { presencia } \\
\text { de pellejos } \\
\text { y tendones } \\
\text { (ligero) }\end{array}$ & $\begin{array}{l}\text { Aspecto al } \\
\text { corte: } \\
\text { presencia de } \\
\text { pellejos y } \\
\text { tendones } \\
\text { (ligero) } \\
\text { Defectos en } \\
\text { la dureza y } \\
\text { dificultad en } \\
\text { el rebanado } \\
\text { (ligero) }\end{array}$ & \begin{tabular}{l} 
Aspecto al \\
corte: \\
presencia de \\
pellejos y \\
tendones \\
(ligero) \\
\\
Defectos en \\
la dureza y \\
dificultad en \\
el rebanado \\
\multicolumn{1}{c}{ (moderado) }
\end{tabular} \\
\hline
\end{tabular}

\subsection{Valoración de los resultados físico-químicos de la Mortadella Novel}

Al valorar la calidad físico-química de la Mortadella Novel, atendiendo a la NRIAL 189:03, para la variante CRD 22 - CCD 33 Lote 6 (tabla 2) se observa que el porcentaje de cloruros se mantuvo dentro de la norma $(1,5 \%$ a $3 \%)$ durante todo el estudio, observándose que comienza a disminuir desde el primer día hasta el día 14, que alcanza la estabilidad, según muestra la figura. 
Figura 1. Comportamiento del \% de cloruros para la Moradella Novel, durante 20 días.

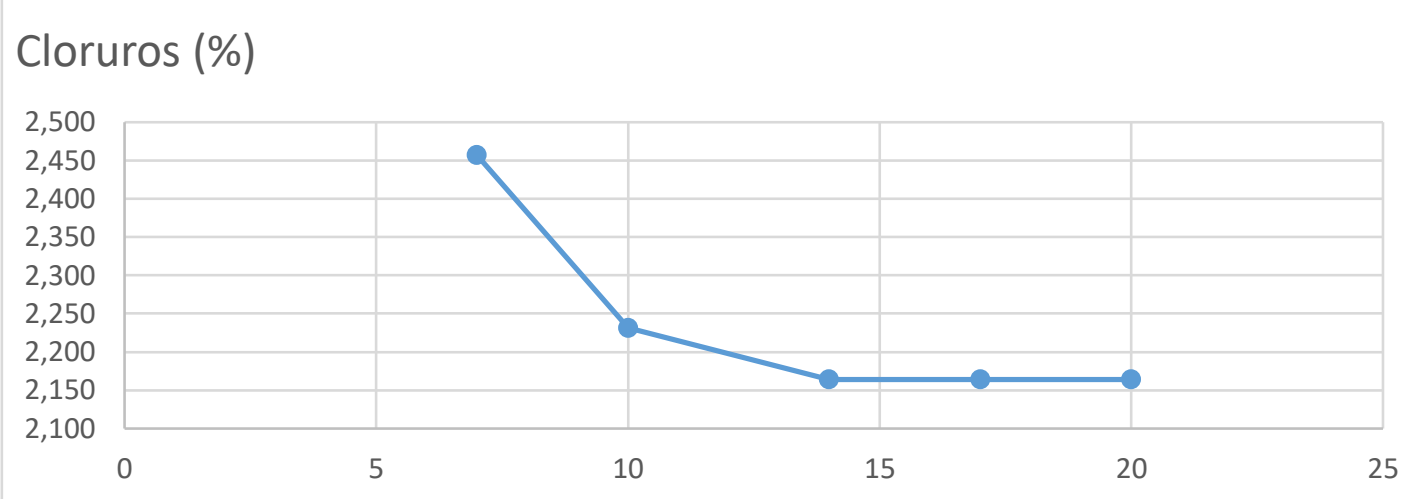

Comportamiento similar tiene la humedad que, según la norma el contenido máximo debe ser de $68 \%$, donde a partir de los 14 días también se mantiene constante y siempre dentro de norma, como se observa en la figura 2.

Figura 2. Comportamiento del \% de humedad para la Moradella Novel, durante 20 días.

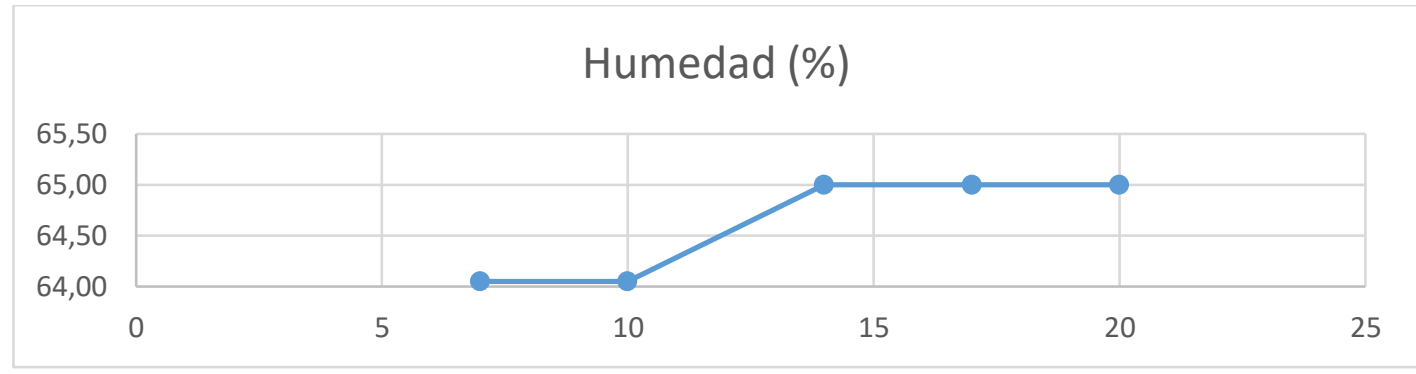

El contenido de nitritos cumple la norma de calidad, lo que realiza su función a la vez que favorece las características del producto. Según Yánez. J, et al, (2005), el contenido de sales en productos embutidos escaldados ayuda a la extracción de proteínas, imparte sabor, mejora la capacidad de retención del agua y además inhibe el crecimiento microbiano. Esta variable se representa gráficamente en la figura 3, durante los 20 días del estudio muestra un comportamiento en ascenso a partir del día 14.

Figura 3. Comportamiento del contenido de Nitritos para la Moradella Novel, durante 20 días.

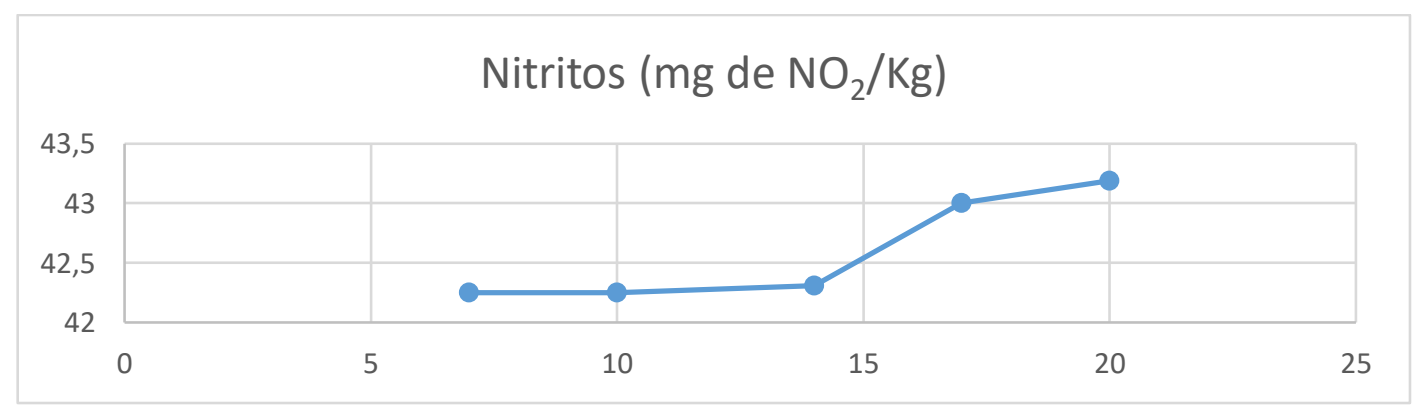


Por otra parte, el pH se mantiene dentro de la norma y constante hasta el día 14, en que comienza a descender (ver fig 4). Al comparar los resultados con la literatura consultada, se encuentra que Viuda-Martos et al. (2011) reporta una reducción en el pH de productos cárnicos durante el almacenamiento, resultante de la acción de bacterias ácido lácticas. Según Yánez. J, et al, (2005) el pH también influye en la capacidad de retención de agua (CRA), (mayor pH, mayor CRA) lo cual favorece que se produzca la emulsificación de la masa a embutir.

Figura 4. Comportamiento del pH para la Moradella Novel, durante 20 días.

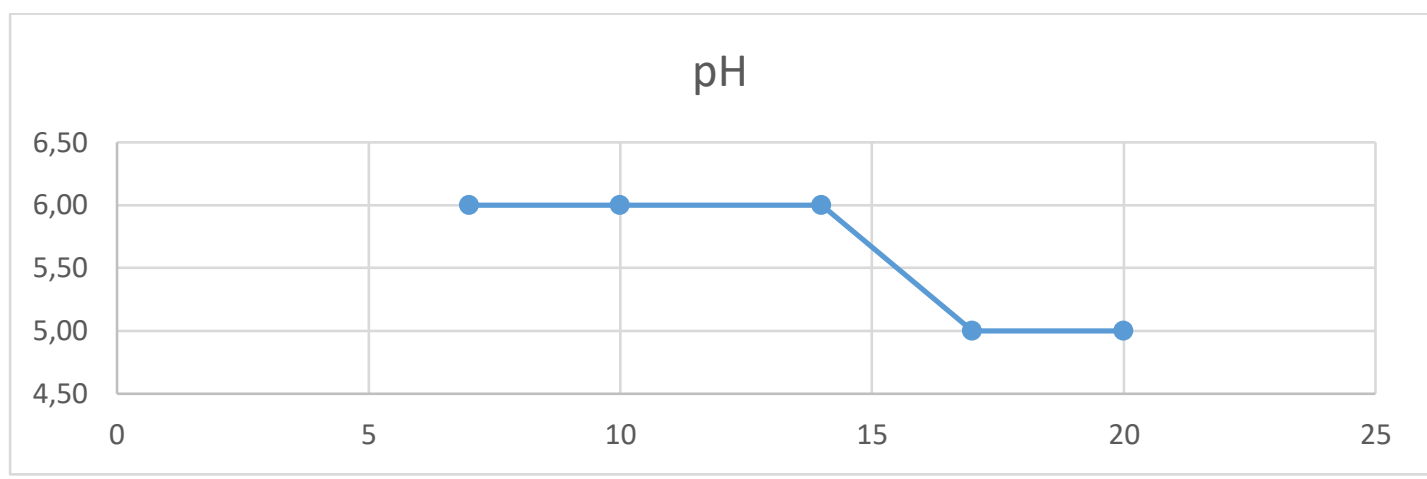

Las variaciones que se han presentado, atendiendo a las variables analizadas, se corresponden con diferencias mínimas entre los valores de cada una, además al comparar con la norma de calidad se observa su cumplimiento, por lo que pudiera pensarse que las variaciones no representan cambios importantes en el producto, sin embargo, se observa que a partir del día 14 existe un punto de viraje para las variables físico-químicas y en cierta medida para las sensoriales. No obstante, en cuanto al análisis microbiológico, el conteo total, de coliformes fecales y de Staphylococcus aureus se mantienen por debajo de lo establecido en las normas de calidad. La presencia de Staphylococcus aureus puede indicar un riesgo potencial para la salud, un número elevado puede indicar la presencia de toxinas termoestables. Este control es importante, dado que muchas especies del género Staphylococcus se consideran habitantes normales del cuerpo humano y el Staphylococcus aureus es el patógeno más destacado. Se requiere un control del mismo debido a que en los humanos, las fosas nasales son los sitios de colonización predominantes, aunque se pueden encontrar células de Staphylococcus aureus en diferentes sitios de la piel, la diseminación puede 
ocurrir por contacto directo o indirecto por fragmentos de piel (Zacarías, Calsin, Aro; 2017). Los resultados para todas las variables se presentan a continuación.

Tabla 2. Resultados de la Mortadella Novel CRD 22 - CCD 33 Lote: 6 FP: 28/1/19.

\begin{tabular}{|c|c|c|c|c|c|c|}
\hline $\begin{array}{l}\text { Tiempo } \\
\text { (días) }\end{array}$ & $\begin{array}{c}\text { Cloruros } \\
(\%)\end{array}$ & $\mathrm{pH}$ & $\begin{array}{l}\text { Humedad } \\
(\%)\end{array}$ & $\begin{array}{l}\text { Nitritos } \\
\text { (mg de } \\
\mathrm{NO}_{2} / \mathrm{kg} \text { ) }\end{array}$ & $\begin{array}{l}\text { Microbiológicos } \\
\quad \text { (ufc/g) }\end{array}$ & Sensoriales \\
\hline 7 & 2,457 & 6,0 & 64,05 & 42,25 & \multirow{5}{*}{$\begin{array}{c}\text { CT: }:<100 \\
\text { CF: }<10 \\
\text { ST: }<100 \\
\text { Salmonella: } \\
\text { negativo }\end{array}$} & 18,0 buena \\
\hline 10 & 2,231 & 6,0 & 64,05 & 42,25 & & 18,0 buena \\
\hline 14 & 2,164 & 6,0 & 65,00 & 42,31 & & 18,0 buena \\
\hline 17 & 2,164 & 5,0 & 65,00 & 43,00 & & $\begin{array}{c}16,4 \\
\text { Aceptable }\end{array}$ \\
\hline 20 & 2,164 & 5,0 & 65,00 & 43,19 & & 12,0 mala \\
\hline
\end{tabular}

CT: Conteo total de microorganismos.

CF: Conteo de coliformes fecales.

ST: Staphylococcus aureus.

CRD: Carne de res deshuesada.

CCD: Carne de cerdo deshuesada.

\subsection{Análisis de varianza}

Para verificar si existen diferencias significativas entre las variantes de mortadella, atendiendo a los resultados químico-físicos se realizó un análisis de varianza, donde se obtuvieron los siguientes resultados.

- $\quad$ Variable cloruros: los resultados se muestran en las tablas 3 y 4.

Tabla 3. Estadística descriptiva

\begin{tabular}{|c|c|c|c|c|c|c|c|}
\hline & Número & Media & DE & CV (\%) & Mín & Máx & Rango \\
\hline CRD 22 - CCD 33 Lote: 6 & 4 & 2,2525 & 0,1422 & 6,313 & 2,16 & 2,46 & 0,3 \\
\hline DE: desviación estándar
\end{tabular}

Tabla 4. Análisis de varianza

\begin{tabular}{|l|c|c|c|c|c|}
\hline Fuente & $\begin{array}{c}\text { Suma de } \\
\text { cuadrados }\end{array}$ & Df & Cuadrado medio & Relación-F & Valor-P \\
\hline Entre grupos & 0,0699 & 3 & 0,0233 & 0,5527 & 0,6568 \\
\hline Dentro grupos & 0,4637 & 11 & 0,0422 & & \\
\hline Total (Corr.) & 0,5336 & 14 & & & \\
\hline
\end{tabular}


Como muestra la tabla 4 , el estadígrafo $F=0,5527$, y el valor de la probabilidad asociada es de 0,6568; valor mayor a 0,05 , por lo que se afirma que no hay diferencias significativas desde el punto de vista estadístico para el porcentaje de cloruros en las cuatro variantes estudiadas, para un nivel de confianza de 95.0\%. Al analizar el resto de las variables en que se comparan todas las variantes desarrolladas en la investigación se observa que no hay diferencias significativas en cuanto a la formulación empleada, lo que corrobora que el tiempo estimado para la vida útil de todas las variantes es el mismo.

Estos resultados se pueden apreciar en las tablas 5,6 y 7 .

Tabla 5. Análisis de varianza variable $\mathrm{pH}$

\begin{tabular}{|l|c|c|c|c|c|}
\hline Fuente & $\begin{array}{c}\text { Suma de } \\
\text { cuadrados }\end{array}$ & Df & $\begin{array}{c}\text { Cuadrado } \\
\text { medio }\end{array}$ & Relación-F & Valor-P \\
\hline Entre grupos & 0,67932 & 3 & 0,22642 & 1,36 & 0,2725 \\
\hline Dentro grupos & 2,00167 & 12 & 0,16681 & & \\
\hline Total (Corr.) & 0,53357 & 15 & & & \\
\hline
\end{tabular}

Tabla 6. Análisis de varianza para los nitritos

\begin{tabular}{|l|c|c|c|c|c|}
\hline Fuente & $\begin{array}{c}\text { Suma de } \\
\text { cuadrados }\end{array}$ & $\boldsymbol{D f}$ & $\begin{array}{c}\text { Cuadrado } \\
\text { medio }\end{array}$ & Relación-F & Valor-P \\
\hline Entre grupos & 631,85 & 3 & 210,617 & 2,15 & 0,1432 \\
\hline Dentro grupos & 1274,05 & 13 & 98,0042 & & \\
\hline Total (Corr.) & 0,5336 & 16 & & & \\
\hline
\end{tabular}

Tabla 7. Análisis de varianza para la humedad

\begin{tabular}{|l|c|c|c|c|c|}
\hline Fuente & $\begin{array}{c}\text { Suma de } \\
\text { cuadrados }\end{array}$ & Df & $\begin{array}{c}\text { Cuadrado } \\
\text { medio }\end{array}$ & Relación-F & Valor-P \\
\hline Entre grupos & 0,05523 & 3 & 0,0181 & 2,75 & 0,0929 \\
\hline Dentro grupos & 0,07345 & 11 & 0,0066 & & \\
\hline Total (Corr.) & 0,12877 & 14 & & & \\
\hline
\end{tabular}

\section{Determinación de vida útil}

Una vez comprobado que las formulaciones tienen el mismo comportamiento, al no existir diferencias significativas entre ellas, atendiendo a los resultados físicoquímicos, se determinaron los modelos matemáticos que rigen el 
comportamiento de cada una de las variables, los que se presentan a continuación:

1. cloruros $=3,073-0,115^{\star}$ días $+0,004^{\star}$ días $^{\wedge} 2$

$$
\text { R-cuadrado }=94,871 \%
$$

2. humedad $=62,415+0,258^{*}$ días $-0,006^{*}$ días $^{\wedge} 2$

$$
\text { R-cuadrado }=84,786 \%
$$

3. nitritos $=42,768-0,131$ *días $+0,008 *$ días $^{\wedge} 2$

R-cuadrado $=91,424 \%$

4. $\mathrm{pH}=5,558+0,124^{*}$ días $-0,008^{*}$ días $^{\wedge} 2$

$$
\text { R-cuadrado }=81,079 \%
$$

En todos los casos los modelos son representativos obteniéndose coeficientes de determinación por encima del 80\%. Estos modelos permiten predecir el momento a partir del cual el producto incumple la norma de calidad establecida y por tanto sus características organolépticas sufrirán deterioro, cesando su vida útil.

En la Mortadella Novel CRD 22 - CCD 33 Lote 6, el pH desciende a valores por debajo de 5,7 a los 15 días, mientras que las variables cloruros, nitritos y humedad se mantienen con valores dentro de lo exigido por la norma de calidad, correspondiéndose este resultado con las evaluaciones sensoriales que se realizaron. La vida útil del producto calculada (14 días) no se corresponde con la planteada por la NEIAL 2660-01: 2017, que es de 7 días, lo que no se corresponde con su durabilidad real.

\section{CONCLUSIONES}

Se estimó el tiempo de vida útil, mediante una variante del método directo, definiéndose que para la Mortadella Novel del Combinado Cárnico Álvaro Barba es de 14 días.

Los modelos matemáticos obtenidos para cada una de las variables físicoquímicas resultan valiosos a la industria pues le permite avizorar el momento en que se afecta la calidad de la mortadella. 
Los resultados de vida útil de la Mortadella Novel en la UEB Álvaro Barba Machado del municipio Colombia, de la provincia Las Tunas contribuyen al logro de la seguridad alimentaria local.

\section{REFERENCIAS}

Aguilera, D (2020). Vida útil de producto marino tipo embutido almacenado en refrigeración, con la adición de un antimicrobiano natural. Proyecto de graduación presentado como requisito parcial para optar al título de Ingeniero en Agroindustria Alimentaria. Escuela Agrícola Panamericana, Zamorano. Honduras.

Cali C, Carolina, G. (2015). Determinación de la concentración residual durante las etapas de nitrito de sodio de elaboración almacenamiento de cinco productos cárnicos de la planta de alimentos piggis embutidos PIGEM Cía. Ltda. y su incidencia sobre el tiempo de vida útil. Universidad Técnica de Ambato. Facultad de Ciencia e Ingeniería en Alimentos. Ecuador.

Da Silva Henrique, G. B. (2016). Evaluación energética en el proceso de obtención de embutidos escaldados. Universidad Central "Marta Abreu" de Las Villas. Trabajo de diploma, Ingeniería Química, Universidad Central " Marta Abreu" de Las Villas, Villa Clara, Cuba.

Llamba, L., y Jackeline, S. (2012). El aseguramiento de la calidad y su incidencia en la producción de embutidos de la Fábrica La Madrileña de la ciudad de Latacunga provincia de Cotopaxi. (Bachelor'sthesis).

NC 189: 03 Carne y productos cárnicos. Mortadella Novel. Especificaciones de calidad. Vigente 2020.

NC ISO 2859-1:2003 Procedimiento de muestreo para la inspección por atributos Parte 1: esquemas de muestreo indexado por el nivel de calidad aceptable (NCA) para la inspección lote a lote. Vigente 2020.

Nuñez de Villavicencio, M; Rodríguez I; Hernández, R: Rodríguez, J.L. (2017) Metodología para la estimación de la vida útil de los alimentos. I. Procedimiento general. Food Science and Technology. Vol. 27, No.1, https://www.researchgate.net/publication/321807907

Peñaherrera, P. (2018). Manual de charcutería enfocado en la elaboración de fiambres y embutidos. Disertación, Universidad de Los Hemisferios, http://dspace.uhemisferios.edu.ec.Quito. Ecuador.

Rubio, A; Español, A; Altolaguirre, J.I; Cabedo, L; Vásquez, A; Iñigo, S; Madrid, E; Fernandez, P; Palop, A. (2019) Documento de orientación para la verificación de estudios de vida útil en relación con Listeria 
monocytogenes en alimentos listos para el consumo. Agencia española de seguridad alimentaria y nutrición. Comisión Institucional. España.

Viuda-Martos, M., Ruiz-Navajas, Y., Fernández-López, J. Pérez-Álvarez, J. A. (2011). Effect of Packaging Conditions on Shelf-Life of Mortadella Made with Citrus Fibre Washing Water and Thyme or Rosemary Essential Oil. Food and Nutrition Sciences (2), 1-10.

Yánez. J; Guerra. M. A; Andújar. G; Santos. R; González. A. M; Pérez. D; Venegas. O; de la Mella. R. M; Ramos. M; Beldarraín. T; y Cepero. Y (2005). Manual de histología, composición y conversión del músculo en carne. Capítulo IX, p. 121-148. Instituto de Investigaciones para la Industria Alimentaria, La Habana, Cuba.

Zacarías P, Calsin M, Aro J.M. (2017) Determinación del tiempo de vida útil de la carne curada de cuy (Cavia porcellus L.) Utilizando diferentes concentraciones de cloruro de sodio. Rev. Investig. Altoandin. Vol 19 № 1: $\quad 53 \quad-\quad 62 . \quad 6$ http://huajsapata.unap.edu.pe/ria http://dx.doi.org/10.18271/ria.2017.255 